\title{
[68Ga]-DOTATATE PET/CT and PET/MRI in the diagnosis and management of esthesioneuroblastoma: illustrative cases
}

\author{
Michelle Roytman, MD, ${ }^{1}$ Andrew B. Tassler, MD, ${ }^{2}$ Ashutosh Kacker, MD, ${ }^{2}$ Theodore H. Schwartz, MD, ${ }^{3}$ Georgiana A. Dobri, MD, ${ }^{4}$ \\ Sara B. Strauss, MD, ${ }^{1}$ Alyssa M. Capalbo, BS, ${ }^{1}$ Rajiv S. Magge, MD, ${ }^{5}$ Marissa Barbaro, MD, ${ }^{5}$ Eaton Lin, MD, ${ }^{1}$ Joseph R. Osborne, MD, PhD, ${ }^{1}$ \\ and Jana Ivanidze, MD, $\mathrm{PhD}^{1}$
}

Departments of ${ }^{1}$ Radiology, ${ }^{2}$ Otolaryngology, Head and Neck Surgery, ${ }^{3}$ Neurological Surgery, ${ }^{4}$ Endocrinology, Diabetes and Metabolism, and ${ }^{5}$ Neuro-Oncology, Weill Cornell Medicine, New York, New York

BACKGROUND Esthesioneuroblastoma (ENB), also known as olfactory neuroblastoma, is a rare sinonasal neuroectodermal malignancy with a slow onset of symptoms, favorable 5-year survival, and a propensity for delayed locoregional recurrence. Current treatment options include resection, adjuvant radiotherapy, and/or chemotherapy; however, because of its rarity and location, determining the optimal treatment for ENB has been challenging.

OBSERVATIONS ENBs strongly express somatostatin receptors (SSTRs), particularly SSTR2, providing a molecular target for imaging and therapy. LESSONS The authors present a case series of ENBs imaged with [68Ga]-DOTATATE PET/MRI and PET/CT and discuss the emerging role of [68Ga]-DOTATATE PET for ENB diagnosis, staging, and treatment response monitoring.

https://thejns.org/doi/abs/10.3171/CASE2058

KEYWORDS esthesioneuroblastoma; olfactory neuroblastoma; [68Ga]-DOTATATE; [177Lu]-DOTATATE; PET; PRRT

Esthesioneuroblastoma (ENB) is a rare sinonasal neuroectodermal malignancy within the heterogeneous family of neuroendocrine tumors $(\mathrm{NETs})^{1}$ with recent evidence supporting an even distribution across all ages. ${ }^{2}$ ENB is thought to originate from stem cells of the olfactory epithelium-derived neural crest known as olfactory placodes. ${ }^{1}$ ENB is phenotypically intermediate between a pure neural neoplasm and a neuroendocrine epithelial tumor and can be easily confused with other "small blue round cell tumors" of the nasal cavity and paranasal sinuses. $^{3}$ ENB has a broad range of differential diagnoses, including sinonasal undifferentiated carcinoma, sinonasal neuroendocrine carcinoma, and small cell carcinoma. Pathological review and ancillary testing are thus critical for differentiating these tumor types. ${ }^{1}$

ENB most commonly presents with nasal obstruction, recurrent epistaxis, headache, facial pain, sinusitis, or anosmia with unilateral symptoms occurring more commonly than bilateral symptoms. ${ }^{1}$ More advanced disease may present with symptoms associated with local extension beyond the nose and paranasal sinuses, such as orbital symptoms or symptoms associated with intracranial involvement of the frontal lobe. ${ }^{1}$ Occasionally, ENB is incidentally detected as an asymptomatic nasal mass, ${ }^{4}$ and, very rarely, paraneoplastic syndromes such as Cushing syndrome and hyponatremia have been reported. ${ }^{5}$ Because of the nonspecific nature of early symptoms, diagnosis is frequently delayed with an average time of $6-12$ months between symptom onset and diagnosis. ${ }^{1}$ Cervical lymph node metastases at initial presentation occur in $5 \%$ of cases, with delayed cervical nodal recurrence in up to $20 \%-25 \%$ of cases. ${ }^{6}$ The cervical level II lymph nodes are the most common site of metastasis; however, involvement of all cervical nodal levels, especially levels I-IV, and the retropharyngeal nodes has been reported. ${ }^{6}$

Staging is determined by the criteria described by Kadish et al.: stage A, tumor restricted to the nasal cavity; stage $B$, tumor involving the nasal cavity and at least one sinus; stage $C$, tumor extending beyond the paranasal cavities; and a modified stage $D$, cervical lymph node metastases. ${ }^{7}$ Other proposed staging systems include the tumor-nodal-metastasis (TNM) system by the American Joint Committee on Cancer and a modified TNM version by Dulguerov. ${ }^{8}$

ABBREVIATIONS CCRT = concurrent chemoradiation therapy; $C T$ = computed tomography; $E N B=$ esthesioneuroblastoma; FDG = fluorodeoxyglucose; LAR = long-acting release; $M I P=$ maximum intensity projection; $M R I=$ magnetic resonance imaging; NET = neuroendocrine tumor; $P E T=$ positron emission tomography; $P R R T=$ peptide receptor radionuclide therapy; $\mathrm{RT}=$ radiotherapy; SSTR = somatostatin receptor; SUV = standardized uptake value.

INCLUDE WHEN CITING Published January 11, 2021; DOI: 10.3171/CASE2058.

SUBMITTED November 1, 2020. ACCEPTED November 6, 2020.

(c) 2021 The authors, CC BY-NC-ND 4.0 (http://creativecommons.org/licenses/by-nc-nd/4.0/). 
Grading is determined by the criteria described by Hyams and Batsakis in which ENB is stratified into four grades ranging from most differentiated (grade I) to least differentiated (grade IV) on the basis of mitotic activity, nuclear pleomorphism, rosette formation, necrosis, disorganized architecture, and sparse fibrillary matrix. ${ }^{9}$ Recent evidence suggests a correlation of the Hyams grading to outcomes, with high-grade (grade III/IV) tumors associated with more aggressive locoregional disease and worse survival outcomes as compared with those associated with low-grade (grade I/II) tumors. Therefore, grading can serve as a prognostic indicator. ${ }^{10}$

ENB is known to have a relatively favorable prognosis, with a 5-year overall survival ranging from $57 \%-93 \%$. The risk of recurrence is highest among those with poor prognostic factors, such as a high Hyams grade, advanced Kadish staging, intracranial extension, and positive resection margins. ${ }^{10}$ Current treatment options include resection, adjuvant radiotherapy (RT), and/or chemotherapy, with recent meta-analysis data favoring surgical treatment followed by RT. ${ }^{11}$ However, because of ENB's rarity, location, and propensity for locoregional recurrence, determining the optimal treatment has been challenging, and no standardized treatment guidelines have been established to date.

Imaging plays a critical role in ENB diagnosis, staging, and management. Computed tomography (CT) and magnetic resonance imaging (MRI) are utilized to assess osseous involvement and soft tissue extension, respectively. ENB appears as a heterogeneous mass with variable enhancement in the upper nasal cavity, cribriform plate, and anterior cranial fossa. ${ }^{12}$ When intracranial extension is present, characteristic peritumoral cysts at the tumor-brain interface can be seen. [18F]-Fluorodeoxyglucose (FDG) positron emission tomography (PET) is also commonly used in patients with advanced disease and for treatment response evaluation. ${ }^{12}$ ENB demonstrates high somatostatin receptor (SSTR) expression, ${ }^{13}$ providing a molecular target for imaging and therapy. [68Ga]-DOTATATE PET allows whole-body imaging of SSTR expression, revolutionizing the detection and characterization of malignancies that overexpress SSTRs, such as ENB. ${ }^{14}$

We present a case series of patients with ENB imaged with [68Ga]DOTATATE PET and discuss the potential role of this emerging imaging modality for this indication.

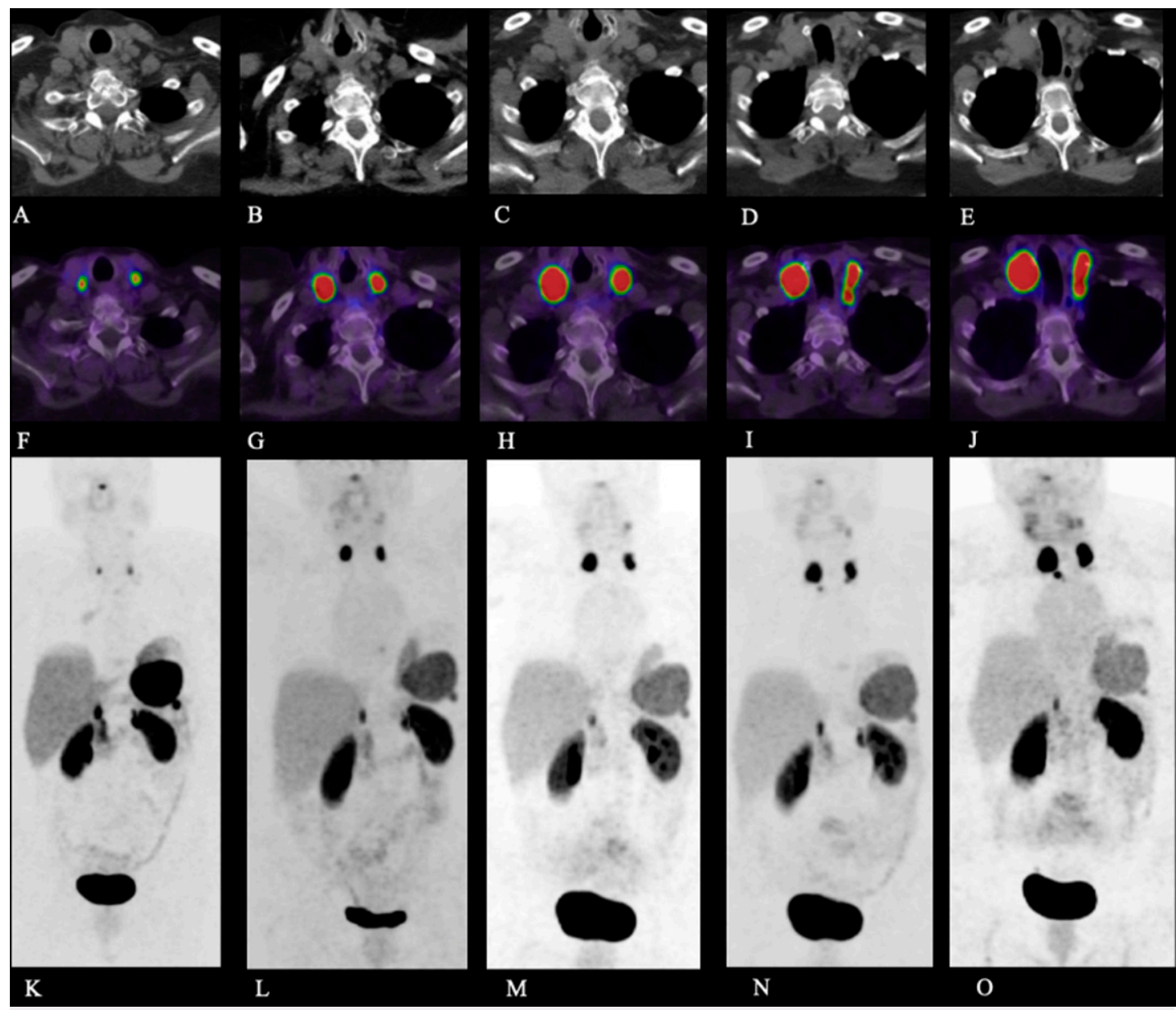

FIG. 1. Case 1. Axial CT (A-E), fused axial [68Ga]-DOTATATE PET/CT windowed at maximum standardized uptake value (SUV) 0-15 (F-J), and [68Ga]-DOTATATE PET maximum intensity projection (MIP) image (K-0) at the following time points post-initial resection: 35 months (A, F, K), 42 months (B, G, L), 48 months (C, H, M), 54 months (D, I, N), and 58 months (E, $\mathrm{J}, \mathbf{0}$ ). The images demonstrate intensely DOTATATE-avid bilateral cervical lymph nodes, compatible with cervical nodal metastases. 


\section{Illustrative Cases}

\section{Case 1}

A 68-year-old woman presented with progressive hyposmia and refractory sinonasal allergic symptoms and was found to have ENB (resected via combined open and endoscopic craniofacial approach; Hyams grade II, Kadish stage C upgraded to D 30 months postinitial resection; multiple positive right level II-V nodes, largest metastatic focus $1.6 \mathrm{~cm})$. The patient received adjuvant concurrent chemoradiation therapy (CCRT) (etoposide, cisplatin; 6900 cGy in 33 fractions) followed by salvage neck dissection. Residual bilateral cervical lymph nodes demonstrated intense avidity on [68Ga]-DOTATATE PET/CT, compatible with cervical nodal metastases. After extensive discussion regarding the goals of care, off-label use of octreotide long-acting release (LAR) $(20,000$ $\mathrm{mcg} I \mathrm{M} \mathrm{q} 4$ weeks) for systemic treatment of metastatic ENB was initiated, and serial imaging with whole-body [68Ga]-DOTATATE PET/CTs was performed every 6 months (Fig. 1). To date, the patient is 68 months post-initial resection and 26 months postinitiation of octreotide LAR treatment. The patient continues to have ongoing evidence of cervical nodal metastases without other signs of systemic metastatic disease.

\section{Case 2}

A 53-year-old woman presented with left periorbital swelling and was found to have ENB (resected via combined anterior craniofacial approach and transcribriform transethmoid approach, followed by left selective neck dissection; Hyams grade IV, Kadish stage D; single left level lla positive node with focal ENB, $4.5 \mathrm{~cm}$ ). The patient received adjuvant CCRT (etoposide, cisplatin; 6600 cGy in 30 fractions) followed by three doses of weekly concurrent cisplatin. Immediate as well as 6-month postoperative MRI, [68Ga]-DOTATATE PET/CT, and [18F]FDG PET/MRI demonstrated postsurgical changes without definitive evidence of recurrent or residual disease. The patient subsequently developed back pain and paresthesias at 9 months post-initial resection with MRI demonstrating leptomeningeal disease in the cerebellum and thoracolumbar spine, which was proven to be metastatic ENB by biopsy. The patient received proton beam craniospinal RT; however, she ultimately died 11 months post-initial resection.

\section{Case 3}

A 64-year-old woman presented with dysphonia and was found to have a 3.6-cm right sphenoethmoid recess lesion, initially presumed to be a nasal polyp, with pathology on polypectomy revealing ENB. Final pathology on subsequent resection confirmed ENB (resected via anterior craniofacial approach; Hyams grade I-II, Kadish stage C). The patient received adjuvant CCRT (cisplatin and carboplatin, terminated after two rounds due to side effects; $6120 \mathrm{cGy}$ in 34 fractions). Followup imaging with CT, MRI, and whole-body [68Ga]-DOTATATE PET/CT demonstrated postsurgical changes without definitive evidence of recurrent or residual disease. Notably, [68Ga]-DOTATATE PET/CT identified an intensely DOTATATE-avid dura-based mass along the right cavernous sinus, most compatible with a meningioma (Fig. 2). To date, the patient is 27 months post-initial resection without evidence of recurrent or residual disease.

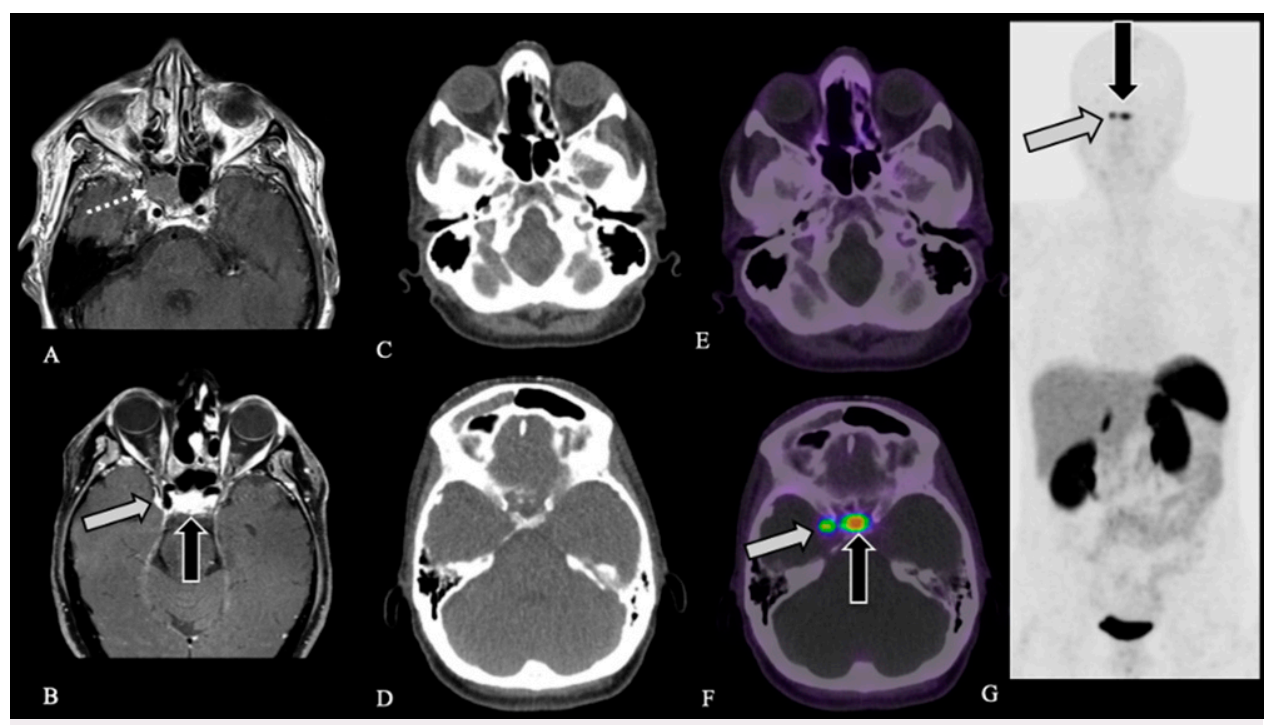

FIG. 2. Case 3. Preoperative axial contrast-enhanced T1-weighted MRI (A) demonstrates patient's initial lesion within the right sphenoid sinus (white dotted arrow), pathology-proven ENB. Postoperative axial contrast-enhanced fat-saturated T1-weighted MRI (B) demonstrates dural-based plaque-like enhancement along the right cavernous sinus (white arrow) and normal enhancement of the pituitary gland (black arrow). Axial CT (C and D) and fused axial [68Ga]-DOTATATE PET/CT windowed at SUV 0-15 (E and F) demonstrate expected postsurgical appearance of the sinuses without evidence of DOTATATE avidity to suggest residual or recurrent disease ( $\mathbf{C}$ and $\mathbf{E}$ ) as well as a focus of intense DOTATATE avidity, SUV 11.0, along the right cavernous sinus (white arrow, $\mathbf{F}$ ), corresponding to the dura-based plaque-like enhancement (B), compatible with a meningioma. Physiological DOTATATE avidity is noted in the pituitary gland (black arrow, F). [68Ga]DOTATATE PET MIP $(\mathbf{G})$ demonstrates normal physiological uptake in the pituitary gland (black arrow) and a focus of DOTATATE avidity corresponding to the right cavernous sinus meningioma (white arrow) without evidence of recurrent or metastatic disease. 


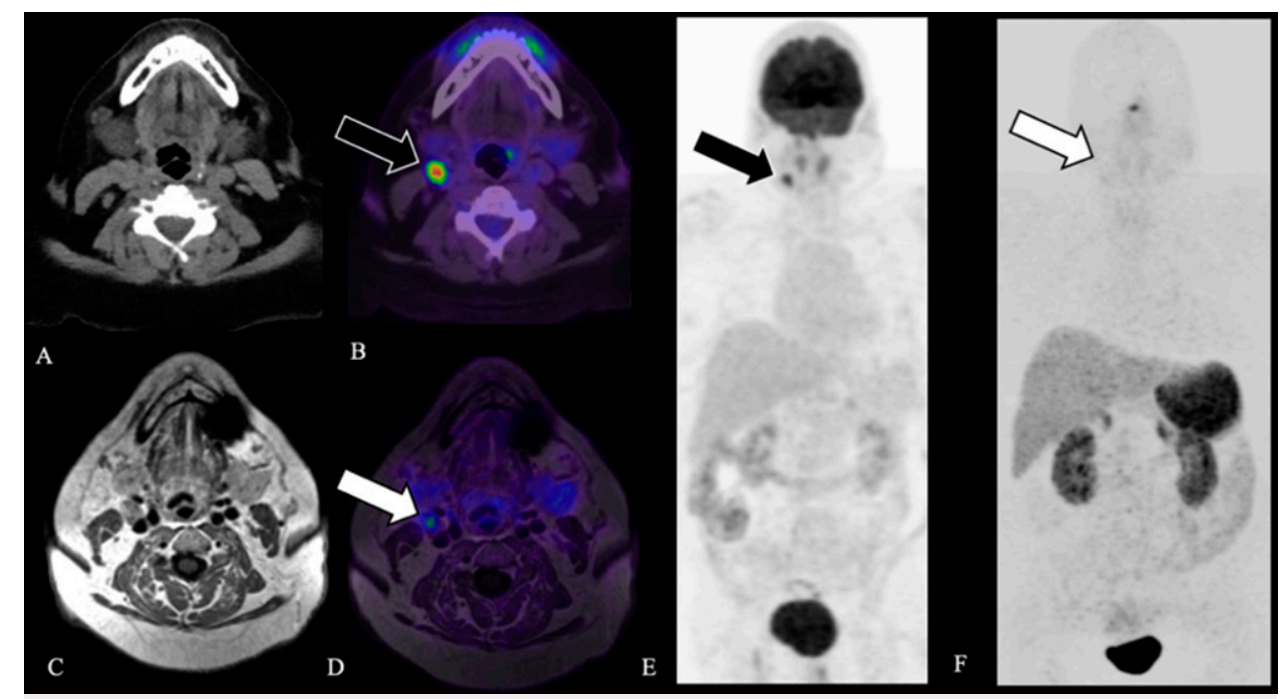

FIG. 3. Case 4. Axial CT (A), fused axial [18F]-FDG PET windowed at SUV 0-15 (B), and [18F]-FDG PET MIP (E) demonstrate a moderately FDG-avid right level lla lymph node, SUV 16.3 (black arrow, B, E). Axial T1weighted MRI (C), fused axial [68Ga]-DOTATATE PET windowed at 0-15 (D), and [68Ga]-DOTATATE PET MIP (F) demonstrate a right level lla lymph node without corresponding avidity (white arrow, $\mathbf{D}, \mathbf{F}$ ), favoring an inflammatory etiology. Surgical excision of the right level lla lymph node confirmed follicular hyperplasia without evidence of malignancy.

\section{Case 4}

A 59-year-old woman presented to an outside hospital with leftsided nasal congestion and was found to have a left posterior nasal cavity mass; biopsy revealed ENB. The patient presented to our institution with ongoing left-sided nasal congestion. CT, MRI, and [18F]FDG PET/CT demonstrated a polypoid mass in the left posterior nasal cavity extending into the sphenoid ostium as well as a moderately FDG- avid dominant right level lla lymph node. The patient underwent fine-needle aspiration sampling of the lymph node, with pathology revealing a benign polymorphous population of lymphoid cells, negative for synaptophysin and chromogranin. The patient underwent resection of the left skull base lesion with final pathology revealing ENB (resected via anterior craniofacial approach; Hyams grade II, Kadish stage B). Postoperative [68Ga]-DOTATATE PET/MRI demonstrated

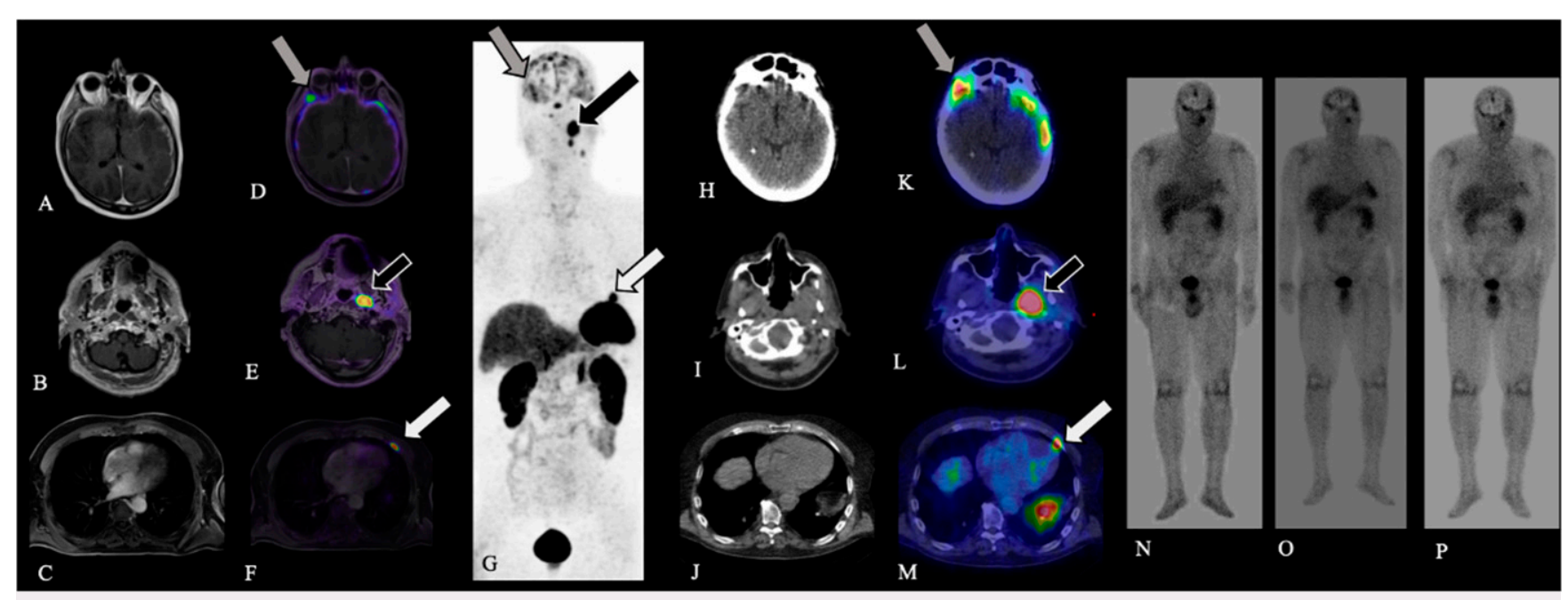

FIG. 4. Case 5. Axial contrast-enhanced T1-weighted MRI (A-C), fused axial [68Ga]-DOTATATE PET windowed at SUV 0-15 (D-F), and [68Ga]DOTATATE PET MIP $(\mathbf{G})$ demonstrate multiple intensely DOTATATE-avid foci involving the dura (A, D, G; gray arrows), left parapharyngeal space (B, E, G; black arrows), and left anterior fourth rib (C, F, G; white arrows), compatible with metastatic ENB. After the administration of [177Lu]-DOTATATE, axial CT (H-J), fused axial [177 Lu]-DOTATATE SPECT windowed at SUV 0-15 (K-M; gray, black, and white arrows), and whole-body planar images obtained at the time of each [177Lu]-DOTATATE treatment (N-P) confirm uptake of [177Lu]-DOTATATE into sites of SSTR-positive metastatic disease. 
postsurgical changes without definitive evidence of recurrent or residual disease. Specifically, the FDG-avid right level lla lymph node was non-DOTATATE avid, favoring an inflammatory, rather than a metastatic, etiology (Fig. 3). The patient opted for surgical excision of the right level Ila lymph node, with pathology revealing follicular hyperplasia in a morphology similar to that of cat-scratch disease without evidence of malignancy. To date, the patient is 10 months post-initial resection without evidence of recurrent or residual disease.

\section{Case 5}

A 65-year-old man presented to an outside hospital with anosmia and was found to have a left nasal cavity mass; a biopsy revealed ENB. The patient presented to our institution for resection with final pathology revealing ENB (resected via combined anterior craniofacial approach and transcribriform transethmoid approach, followed by a left selective neck dissection; Hyams grade II, Kadish stage $C$ upgraded to $D$ 33 months post-initial resection; multiple positive left level II-IV nodes, largest metastatic focus $3.2 \mathrm{~cm}$ with extranodal extension). The patient underwent bilateral neck RT (6000 cGy in 30 fractions) and was followed with serial imaging. [68Ga]-DOTATATE PET/CT 68 months post-initial resection demonstrated an intensely DOTATATE-avid left parapharyngeal space mass, which was proven to be ENB by biopsy, as well as a focus of intense DOTATATE avidity within the left 4 th rib and multiple foci of dura-based DOTATATE avidity along the inner table of the calvaria (Fig. 4). Initiation of treatment was delayed due to hospitalization related to new-onset seizures. Subsequent [68Ga]DOTATATE PET/CT 74 months post-initial resection demonstrated increased size and avidity of multifocal ENB metastases, and systemic treatment with [177Lu]-DOTATATE and octreotide LAR was initiated 76 months post-initial resection. To date, the patient has received three of four doses of [177Lu]-DOTATATE without known adverse events 83 months post-initial resection. Evaluation for treatment response will be performed at the completion of [177Lu]-DOTATATE treatment (7.4 GBq [200 mCi] as an intravenous infusion over 30 minutes every 8 weeks for a total of 4 doses).

\section{Discussion \\ Observations}

The clinical relevance and potential application of SSTR-targeted imaging for ENB diagnosis, staging, and surveillance was evaluated in 2005 utilizing [111/n]-DTPA-D-Pheoctreotide ([111ln]-pentetreotide) with histological correlation. ${ }^{13}$ Resected tumor specimens demonstrated ubiquitous high SSTR2 expression on the protein and mRNA levels as well as high scintigraphic [111ln]-pentetreotide activity, with variable expression of other SSTR subtypes. This study has served as the foundation for SSTR-targeted imaging in ENB.

Recent advances have allowed for the transition from scintigraphy to PET imaging with [68Ga]-labeled SSTR-targeted radiotracers. Various [68Ga]-labeled somatostatin analogs have entered clinical practice, including DOTATATE, DOTATOC, and DOTANOC, each varying in their short chain peptide hormone analog bound to the [68Ga]-labeled radiotracer and DOTA chelator. Advantages of SSTR2targeted PET imaging include improved resolution, improved accuracy due to 100 -fold increased SSTR2 binding (compared with pentetreotide), decreased radiation dose, decreased cost, and increased patient convenience due to the shorter half-life of [68Ga], obviating the need for 24-hour delayed imaging. ${ }^{15}$

Within our cohort, [68Ga]-DOTATATE PET demonstrated utility in its ability to evaluate treatment response (cases 1 and 5), identify the osseous and soft tissue metastatic burden (case 5), and distinguish metastatic from inflammatory adenopathy (case 4). In particular, case 4 highlights the superiority of [68Ga]-DOTATATE PET as compared with [18F]-FDG PET in identifying a nodal metastasis, with excisional biopsy

TABLE 1. Clinical and demographic characteristics of the study population, including presenting symptoms, grade, stage, treatment, and clinical outcome to date

\begin{tabular}{|c|c|c|c|c|c|c|c|}
\hline $\begin{array}{l}\text { Case } \\
\text { No. }\end{array}$ & Gender & $\begin{array}{c}\text { Age at } \\
\text { Diagnosis } \\
\text { (yrs) }\end{array}$ & $\begin{array}{l}\text { Presenting } \\
\text { Symptom }\end{array}$ & $\begin{array}{c}\text { Grade } \\
\text { (Hyams) }\end{array}$ & Stage (Kadish) & Treatment & Clinical Outcome \\
\hline 3 & $\mathrm{~F}$ & 64 & Dysphonia & $|-| \mid$ & C & $\begin{array}{l}\text { Surgery \& adjuvant } \\
\text { RT (6120 cGy } \\
\text { in } 34 \text { fractions) }{ }^{*}\end{array}$ & $\begin{array}{c}\text { Deceased ( } 11 \text { mos post-initial surgery) } \\
\text { no evidence of disease ( } 27 \text { mos } \\
\text { post-initial surgery) }\end{array}$ \\
\hline 4 & $\mathrm{~F}$ & 59 & $\begin{array}{l}\text { Unilateral nasal } \\
\text { congestion/ } \\
\text { obstruction }\end{array}$ & II & B & Surgery & $\begin{array}{c}\text { No evidence of disease (10 mos } \\
\text { post-initial surgery) }\end{array}$ \\
\hline
\end{tabular}

\footnotetext{
* Terminated chemotherapy due to side effects.
} 
confirming the diagnostic accuracy. It is, however, important to note that low-level [68Ga]-DOTATATE avidity can be seen with infection or inflammation ${ }^{16}$ and may confound interpretation, particularly if identified within the resection cavity in the immediate postoperative setting. Further investigation will be required to determine the optimal time period to postoperatively image ENB.

Case 2 highlights the importance of continued clinical and imaging follow-up because ENB has the potential for an aggressive course, particularly those of higher grade and stage. This patient developed leptomeningeal metastases shortly after multiple negative multimodal imaging examinations and ultimately succumbed to her disease. Patients with ENB must receive close clinical and imaging follow-up, with prompt evaluation of new neurological symptoms.

Case 3 highlights the consideration that [68Ga]-DOTATATE PET is highly sensitive, but not specific, for ENB. Therefore, [68Ga]DOTATATE avidity will be present for any lesion demonstrating SSTR2 expression (e.g., meningiomas), and the presence of [68Ga]DOTATATE avidity must be interpreted in conjunction with anatomical MRI/CT features and clinical context. Physiological [68Ga]-DOTATATE avidity is seen in organs with a high number of SSTR2s, such as the pituitary gland, spleen, adrenal glands, liver, kidneys, and urinary bladder. ${ }^{15}$ Potential sources of misinterpretation must be considered, such as the presence of splenules, focal increased uptake in the uncinate process of the pancreas, infection, inflammation, or the presence of other SSTR-positive lesions. The possibility of a falsenegative result must also be considered, with known causes including high-grade NETs without SSTR expression, chemotherapeutic agents modifying SSTR expression, and increased physiological uptake concealing mild uptake elsewhere in the body. ${ }^{15}$

Beyond its application in imaging, the ubiquitous expression of SSTR2 in ENB tumor tissue supports the use of SSTR-based peptide receptor radionuclide therapy (PRRT) for ENB. PRRT is currently approved by the US Food and Drug Administration for treatment of unresectable gastroenteropancreatic NETs (GEP-NETs) based on results of the Neuroendocrine Tumors Therapy Phase 3 trial. In this multicenter randomized trial, patients with GEP-NETs treated with PRRT demonstrated markedly longer progression-free survival, a significantly higher response rate, and an overall survival benefit. ${ }^{17}$

In the only reported case series $(n=7)$ of patients with Kadish stage $\mathrm{D}$ disease and prior conventional multimodality treatment who underwent PRRT, ${ }^{18,19}$ 6/7 patients exhibited partial response or stable disease, with a median progression-free survival of 17 months (range 0-30 months) and median overall survival of 32 months (range 4-53 months). Within this small cohort of unresectable, locally advanced, or metastatic ENBs, PRRT demonstrated promising efficacy and moderate survival duration, and these findings have been supported in other isolated case reports. ${ }^{20}$ Larger cohort studies evaluating survival benefit, disease control, and quality of life of ENB patients treated with PRRT are needed; however, [68Ga]-DOTATATE PET and [177Lu]-DOTATATE PRRT continue to represent promising diagnostic and treatment options in this population.

\section{Lessons}

We present a series of five patients (Table 1) with ENB imaged with [68Ga]-DOTATATE PET/CT and/or PET/MRI, demonstrating its utility in the diagnosis, staging, and treatment-response monitoring of patients with ENB. This imaging modality should be a diagnostic consideration when managing patients with ENB. Limitations of these findings include the small cohort size, limited follow-up period, and lack of pathological confirmation of presumed negative disease in the absence of [68Ga]-DOTATATE avidity.

\section{References}

1. Abdelmeguid AS. Olfactory neuroblastoma. Curr Oncol Rep. 2018;20(1):7.

2. Bell D. Sinonasal neuroendocrine neoplasms: current challenges and advances in diagnosis and treatment, with a focus on olfactory neuroblastoma. Head Neck Pathol. 2018;12(1):22-30.

3. Thompson LDR. Olfactory neuroblastoma. Head Neck Pathol. 2009;3(3):252-259.

4. Ward PD, Heth JA, Thompson BG, et al. Esthesloneuroblastoma: results and outcomes of a single institution's experience. Skull Base. 2009;19(2):133-140.

5. Chung YS, Na M, Ku CR, et al. Adrenocorticotropic hormonesecreting esthesioneuroblastoma with ectopic Cushing's syndrome. Yonsei Med J. 2020;61(3):257-261.

6. Banuchi VE, Dooley L, Lee NY, et al. Patterns of regional and distant metastasis in esthesioneuroblastoma. Laryngoscope. 2016; 126(7):1556-1561.

7. Kadish S, Goodman M, Wang CC. Olfactory neuroblastoma. A clinical analysis of 17 cases. Cancer. 1976;37(3):1571-1576.

8. Joshi RR, Husain Q, Roman BR, et al. Comparing Kadish, TNM, and the modified Dulguerov staging systems for esthesioneuroblastoma. J Surg Oncol. 2019;119(1):130-142.

9. Hyams V, Batsakis JML. Tumors of the upper respiratory tract and ear. Armed Forces Institute of Pathology fascicles, 2nd series. Washingt Am Regist Pathol Press; 1988.

10. Bell D, Saade R, Roberts D, et al. Prognostic utility of Hyams histological grading and Kadish-Morita staging systems for esthesioneuroblastoma outcomes. Head Neck Pathol. 2015;9(1): 51-59.

11. Dulguerov P, Allal AS, Calcaterra TC. Esthesioneuroblastoma: a meta-analysis and review. Lancet Oncol. 2001;2(11):683-690.

12. Dublin $A B$, Bobinski $M$. Imaging characteristics of olfactory neuroblastoma (esthesioneuroblastoma). J Neurol Surg B Skull Base. 2016;77(1):1-5.

13. Rostomily RC, Elias M, Deng M, et al. Clinical utility of somatostatin receptor scintigraphic imaging (octreoscan) in esthesioneuroblastoma: a case study and survey of somatostatin receptor subtype expression. Head Neck. 2006;28(4):305-312.

14. Hofman MS, Lau EWF, Hicks RJ. Somatostatin receptor imaging with $68 \mathrm{Ga}$ DOTATATE PET/CT: clinical utility, normal patterns, pearls, and pitfalls in interpretation. Radiographics. 2016;35(2): $500-516$

15. Ivanidze J, Roytman M, Sasson A, et al. Molecular imaging and therapy of somatostatin receptor positive tumors. Clin Imaging. 2019;56:146-154.

16. Shastry M, Kayani I, Wild D, et al. Distribution pattern of 68Ga-DOTATATE in disease-free patients. Nucl Med Commun. 2010;31(12):1025-1032.

17. Strosberg J, Wolin E, Chasen B, et al. Health-related quality of life in patients with progressive Midgut Neuroendocrine tumors treated with ${ }^{177}$ Lu-dotatate in the Phase III NETTER-1 Trial. J Clin Oncol. 2018;36(25):2578-2584.

18. Sabongi JG, Gonçalves MCP, Alves CDC, et al. Lutetium 177DOTA-TATE therapy for esthesioneuroblastoma: A case report. Exp Ther Med. 2016;12(5):3078-3082.

19. Hasan OK, Ravi Kumar AS, Kong G, et al. Efficacy of peptide receptor radionuclide therapy for esthesioneuroblastoma. J Nucl Med. 2020;61(9):1326-1330.

20. Schneider JR, Shatzkes DR, Scharf SC, et al. Neuroradiological and neuropathological changes after 177lu-octreotate peptide receptor radionuclide therapy of refractory esthesioneuroblastoma. Oper Neurosurg (Hagerstown). 2018;15(6):100-109. 


\section{Disclosures}

Dr. Ivanidze received grants from Advanced Accelerator Applications, A Novartis Company, for this work and receives grants from GE Healthcare for activities outside of this work. Dr. Lin received grants from Novartis for this work. Dr. Roytman reports a 2019 RSNA Resident Research Grant (RR\#1962).

\section{Author Contributions}

Conception and design: Ivanidze, Roytman, Schwartz, Lin, Osborne. Acquisition of data: Ivanidze, Roytman, Tassler, Kacker, Schwartz, Dobri, Strauss, Capalbo, Magge, Lin. Analysis and interpretation of data: Ivanidze, Roytman, Schwartz, Strauss. Drafting the article: Ivanidze, Roytman, Schwartz, Lin, Osborne. Critically revising the article: all authors. Reviewed submitted version of manuscript: Ivanidze, Roytman, Tassler, Kacker, Strauss, Capalbo, Barbaro, Lin,
Osborne. Approved the final version of the manuscript on behalf of all authors: Ivanidze. Statistical analysis: Ivanidze. Administrative/ technical/material support: Ivanidze, Schwartz, Capalbo, Osborne. Study supervision: Ivanidze.

\section{Supplemental Information}

\section{Previous Presentations}

Case 1 was presented as an oral case excerpta at the Eastern Neuroradiological Society 30th Annual Meeting held in Southampton, Bermuda, in 2018.

\section{Correspondence}

Jana Ivanidze: Weill Cornell Medicine, New York, NY. jai9018@ med.cornell.edu. 